\title{
Genome-wide association study reveals candidate genes for flowering time in cowpea (Vigna unguiculata [L.] Walp)
}

1 Dev Paudel ${ }^{1}$, Rocheteau Dareus ${ }^{1}$, Julia Rosenwald², María Muñoz-Amatriaín², Esteban F.

$2 \operatorname{Rios}^{1 *}$

$3 \quad{ }^{1}$ Agronomy Department, University of Florida, Gainesville, Florida, USA

$4 \quad{ }^{2}$ Soil \& Crop Sciences Department, Colorado State University, Fort Collins, Colorado, USA

5 * Correspondence:

6 Corresponding Author

7 estebanrios@ufl.edu

8 Keywords: GWAS, legume, breeding, SNP, gene

9 Abstract

10 Cowpea (Vigna unguiculata [L.] Walp., diploid, $2 n=22$ ) is a major crop used as a protein source for

11 human consumption as well as a quality feed for livestock. It is drought and heat tolerant and has

12 been bred to develop varieties that are resilient to changing climates. Plant adaptation to new climates

13 and their yield are strongly affected by flowering time. Therefore, understanding the genetic basis of

14 flowering time is critical to advance cowpea breeding. The aim of this study was to perform genome-

15 wide association studies (GWAS) to identify marker trait associations for flowering time in cowpea

16 using single nucleotide polymorphism (SNP) markers. A total of 367 accessions from a cowpea mini-

17 core collection were evaluated in Ft. Collins, CO in 2019 and 2020, and 292 accessions were

18 evaluated in Citra, FL in 2018. These accessions were genotyped using the Cowpea iSelect 
19 Consortium Array that contained 51,128 SNPs. GWAS revealed seven reliable SNPs for flowering

20 time that explained 8-12\% of the phenotypic variance. Candidate genes including FT, GI, CRY2,

21 LSH3, UGT87A2, LIF2, and HTA9 that are associated with flowering time were identified for the

22 significant SNP markers. Further efforts to validate these loci will help to understand their role in

23 flowering time in cowpea, and it could facilitate the transfer of some of this knowledge to other

24 closely related legume species.

\section{Introduction}

26 Cowpea (Vigna unguiculata [L.] Walp., diploid, $2 n=22$ ) is a major crop grown worldwide for food

27 and nutritional security (Lonardi et al., 2019). It is well adapted to hot, semi-arid environments, and

28 is highly drought and heat tolerant (Hall et al., 1997). Annual cowpea production is estimated at 7

29 million tons of dry grain harvested on about 14 million hectares worldwide (Singh, 2020). It is grown

30 in over two-thirds of the developing world where it is a major source of protein for human

31 consumption, fodder for livestock (Tarawali et al., 1997), and provides ecosystem services as a cover

32 crop to enhance soil fertility and suppresses weeds (Martins et al., 2003; Rodrigues et al., 2013).

33 Well-fed livestock provide meat, milk, traction, and manure that contributes towards the

34 sustainability of farming systems (Kristjanson et al., 2001). More importantly, cowpea forms a

35 symbiotic association with root nodulating bacteria and fixes nitrogen directly to the soil (Martins et

36 al., 2003). This biological nitrogen fixation improves crop growth and grain production without

37 increasing production costs associated with application of nitrogen fertilizers. Crop rotation including

38 cowpea also helps to decrease instances of Striga hermonthica, a parasitic weed of cereals (Berner et

39 al., 1996).

40 Plant breeders exploit germplasm diversity to generate phenotypic variation for traits under selection,

41 primarily for those influenced by climate variability (Brummer et al., 2011). Therefore, genetic and 
phenotypic characterization of germplasm collections is critical to warrant the development of

43 resilient varieties that will sustain production under future scenarios of climate change. Previous

44 cowpea genetic diversity study using a GoldenGate genotyping assay consisting of 1,536 single

45 nucleotide polymorphisms (SNP)s on 442 cowpea landraces revealed the presence of two major gene

46 pools in cultivated cowpea in Africa (Huynh et al., 2013). A diverse set of 768 cultivated cowpea

47 genotypes from 58 countries were also studied using SNP markers from genotyping by sequencing

48 (GBS) that divided the population into 3 gene pools (America, Africa, and Central West Asia)

49 (Xiong et al., 2016). Lastly, a set of 368 cultivated cowpeas genotyped with 51,128 SNPs revealed

50 six major subpoulations (Muñoz-Amatriaín et al., 2021). Large collections of diverse cowpea

51 accessions are conserved in the International Institute of Tropical Agriculture (IITA) ( 15,000

52 accessions), United States Department of Agriculture - Genetic Resources Information Network

53 (USDA-GRIN) (7,737 accessions), and University of California, Riverside, USA ( 6,000

54 accessions). The large number of conserved accessions in gene bank precludes their direct utilization

55 in a breeding program owing to resource limitations in characterizing the whole collection.

56 Therefore, a mini-core collection consisting 298 lines from the IITA collection were genotyped based

57 on genotyping by sequencing (GBS) using 2,276 SNP markers in order to make the characterization

58 and utilization of the germplasm more practical (Fatokun et al., 2018). Similarly, another mini-core

59 collection, the 'UCR Minicore', consisting of 368 accessions that included landraces and breeding

60 materials from 50 countries was also developed (Muñoz-amatriaín et al.) and genotyped using a

61 publicly available Cowpea iSelect Consortium Array (Muñoz-Amatriaín et al., 2017). This array

62 consists of 51,128 assays developed from sequencing 36 diverse accessions and was released to

63 facilitate easy high-throughput genotyping in cowpea (Muñoz-Amatriaín et al., 2017). While

64 progress has been made through conventional breeding in cowpea, the availability of these new

65 molecular genetic tools enables application of modern breeding strategies for cowpea improvement

66 (Gupta et al., 2014). 
67 Flowering time is a key player in plant adaptation and is an important phenological trait to breed for

68 because agronomic traits such as plant growth, plant height, and grain quality depend on the timing

69 of flowering (Durand et al., 2012; González et al., 2016). Early flowering plants could mature earlier

70 and help plants to avoid terminal drought stress (Kumar and Abbo, 2001). Crop legumes show large

71 variation in flowering time, which has aided their improvement using selection and breeding (Weller

72 and Ortega, 2015). High heritability estimates for days to flowering are reported in legumes, ranging

73 from broad sense heritability on an entry-mean basis of 0.77 to 0.95 in soybean (Zhang et al., 2015;

74 Mao et al., 2017), 0.38-0.75 in alfalfa (Adhikari et al., 2019), and narrow-sense heritability of 0.63 -

750.86 in cowpea (Ishiyaku et al., 2005). In many species, flowering is induced in response to day

76 length. Different flowering responses are categorized as short-day, long-day, intermediate-day, or

77 day-neutral based on the day length requirement to induce flowering (Bastow and Dean, 2002). Most

78 cowpea genotypes are short-day, in which flowering is favored by day lengths shorter than the

79 corresponding nights, while some genotypes are insensitive to a wide range of photoperiods

80 (Summerfield and Roberts, 1985). Warmer temperatures can hasten the appearance of flowers in both

81 daylength-sensitive and insensitive genotypes (Summerfield and Roberts, 1985). The critical

82 photoperiod for cowpea at $27^{\circ} \mathrm{C}$ was reported to be between 12 and $13 \mathrm{~h} \mathrm{day}^{-1}$ (Craufurd et al., 1996).

83 Owing to the importance of flowering time in cowpea, studies in the past have focused on identifying

84 quantitative trait locus (QTL) using SNP and simple sequence repeat (SSR) markers in recombinant

85 inbred lines (RIL). Five QTLs related to time of flower opening and three QTLs related to days to

86 flower were identified in a RIL population of 524B x 219-01 using SSR markers (Andargie et al.,

87 2013). SNP and SSR markers were utilized in another RIL population of ZN016 x ZJ282 to identify

88 QTLs for days to first flowering, nodes to first flower, leaf senescence, and pod number per plant (Xu

89 et al., 2013). One major QTL and few minor QTLs were found to dominate each of the four traits

90 with three to four QTLs controlling individual traits. Other studies aimed at deciphering the genetics 
91 of flowering time in cowpea have proposed one-gene (Sène, 1967) and seven-gene (Ishiyaku et al.,

92 2005) models to control flowering. Recent advances in genomic technologies has enabled a better

93 understanding of the genetic basis of variation using GWAS, as it can be used for identification and

94 high resolution mapping of useful genetic variability from germplasm sets that have resulted from

95 many rounds of historical recombination (Yu and Buckler, 2006). GWAS studies have been reported

96 in cowpea for pod length (Xu et al., 2017), root architecture (Burridge et al., 2017), black seed coat

97 color (Herniter et al., 2018), seed weight, length, width, and density (Lo et al., 2019), and plant

98 productivity traits and flowering time (Muñoz-Amatriaín et al., 2021). The study of Muñoz-

99 Amatriaín et al. (2021) evaluated flowering time in five different environments in Nigeria and

100 California, most of which were short-day environments.

101 Existing genetic diversity of cowpea needs to be assessed in order to strengthen breeding programs

102 for developing high yielding dual-purpose cultivars with good grain and fodder yields. In this study,

103 we phenotyped the UCR Minicore in Ft. Collins, CO and Citra, FL and performed GWAS for days to

104 flowering; and identified candidate genes related to flowering time in cowpea.

\section{Materials and methods}

\subsection{Germplasm, Site Description, and Experimental Design}

107 A total of 367 accessions from the cowpea UCR Minicore (Muñoz-Amatriaín et al., 2021) were

108 planted in Ft. Collins, Colorado $\left(40.6553^{\circ} \mathrm{N},-104.9966^{\circ} \mathrm{W}\right)$ on June 17,2019 . This collection

109 includes landraces and breeding materials from 50 countries. Seeds from each accession were planted

110 in $6.4 \mathrm{~m}$ rows with $0.9 \mathrm{~m}$ alley and 50 seeds per plot. The experiment was set up as row/column

111 design with one replication and augmented representation of two control lines (CB5 and CB46). Plots

112 were irrigated at the rate of $25.4 \mathrm{~mm}$ every week. The experiment was repeated in 2020 when the

113 plots were planted on June 5, 2020. 
114 A total of 292 cowpea accessions from the cowpea mini-core collection that had mature pods in

115 October 2017 were selected from a UC-Riverside field location and planted in the field at the Plant

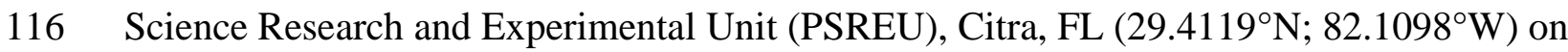

117 September 7, 2018 (Dareus et al., 2021). The soil was a Chipley sand (thermic, coated Aquic

118 Quartzipsamments) with a pH of 6.9 and characterized by high $\mathrm{P}_{2} \mathrm{O}_{5}$ content, and low $\mathrm{K}_{2} \mathrm{O}, \mathrm{S}$, and

$119 \mathrm{Mg}$ content. Seeds from each accession were planted in single row of 10 plants per plot, and the

120 experiment was set up as a row/column design with two replications and augmented representation of

121 ten control lines. Each experimental unit $(3 \mathrm{~m} \mathrm{x} 0.6 \mathrm{~m})$ consisted of ten plants manually seeded and

122 spaced at $0.3 \mathrm{~m}$ within row and $0.6 \mathrm{~m}$ between row spacing (Dareus et al., 2021).

\section{$123 \quad 2.2 \quad$ Phenotypic trait and analyses}

124 Days to flowering in Colorado was taken as the number of days from seeding to first time $50 \%$ of the

125 plants of a given accession flowered. In Florida, days to flowering was monitored every two days,

126 and days to first flowering was counted as the number of days from planting to the day when at least

$12710 \%$ of the plants in the experimental unit exhibited flowers. Descriptive analysis, and analysis of

128 variance (ANOVA) were conducted in the R statistical package (R Development Core Team, 2020).

129 Variance components were estimated using mixed linear models in ASReml-R v.4 (Butler et al.,

130 2017). Best linear unbiased estimate (BLUE) and best linear unbiased prediction (BLUP) for each

131 trait was extracted for every accession using ASREML-R (Butler et al., 2017). Broad-sense

132 heritability $\left(H^{2}\right)$ was calculated using variance components by the formula $\mathrm{H}^{2}=\mathrm{VG} /(\mathrm{VG}+\mathrm{VE})$, where

133 VG represents genetic variance and VE represents the residual variance.

\section{$134 \quad 2.3 \quad$ SNP genotyping}


135 SNP genotyping is previously described (Muñoz-Amatriaín et al., 2017). Briefly, total genomic DNA

136 from single plants was extracted from dried leaves using Plant DNeasy (Qiagen, Germany) and

137 genotyped using the Cowpea iSelect Consortium Array that contained 51,128 SNPs. SNPs were

138 called using GenomeStudio software V.2011.1 (Illumina, Inc. San Diego, CA) and the physical

139 positions of the SNPs were determined by using the IT97K-499-35 reference genome v1.0 (Lonardi

140 et al., 2019).

\section{$141 \quad 2.4 \quad$ Genome-wide association study}

142 Marker trait association (MTA) using all SNP markers were evaluated based on the BLUE values for

143 days to flower. A minor allele frequency (MAF) threshold of 5\% was used to remove rare variants

144 and avoid false-positive associations. Multiple algorithms were applied for GWAS. For all SNP loci

145 and phenotypic data, we applied the generalized linear model (GLM) and mixed linear model (MLM)

146 implemented in GAPIT (Lipka et al., 2012). Further, GWAS was conducted using Fixed and random

147 model Circulating Probability Unification (FarmCPU) algorithm that takes into account the

148 confounding problem between covariates and test marker by using both Fixed Effect Model (FEM)

149 and a Random Effect Model (REM) (Liu et al., 2016). GWAS was also conducted using BLINK that

150 uses Bayesian Information Content (BIC) in a fixed effect model and replaces the bin approach used

151 in FarmCPU with linkage disequilibrium (Huang et al., 2018b). Six principal components from

152 GAPIT were used as covariates to control for population structure and manhattan plots were drawn

153 using package qqman (Turner, 2014) in R statistical package (R Development Core Team, 2020).

\section{$154 \quad 2.5 \quad$ Candidate gene identification}

155 For candidate gene identification, the reference genome of cowpea IT97K-499-35 v1.0 (Lonardi et

156 al., 2019) and the corresponding annotation (Vunguiculata_469_v1.1.annotation_info.csv) and gff

157 file (vigna_genesv1_1_gff.csv) were used. A region of $270 \mathrm{~kb}$ above and below the significant SNPs 
was further evaluated and gene models were extracted to identify candidate genes. Orthologs of these

159 genes on Arabidopsis were identified and functionally characterized using TAIR database

160 (www.arabidopsis.org) and their molecular functions were elucidated. Gene models whose Gene

161 Ontology (GO) function was related to flowering were selected as candidate genes and their function

162 was searched in the literature.

\section{Results}

\section{$164 \quad 3.1 \quad$ Phenotypic Analysis}

165 There was a significant variation in days to flower in all the datasets evaluated (Table 1, Figure 1). In

166 Colorado in 2019, the average days to flowering was 75 days and in 2020 it was 72 days. Days to

167 flowering was much earlier in Florida. In Florida in 2018, the average days to flowering was 41 days

168 with a range of 32-69 days. Range of flowering was also shorter in Florida as compared to Colorado.

169 Broad-sense heritability for flowering time ranged from 0.72-0.95 for the three studies. Pearson's

170 correlation between the BLUEs for the three datasets were positive $(0.44-0.81)(p<0.05)$ showing

171 that early flowering lines in Florida also flowered early in Colorado in both years.

\section{$172 \quad 3.2 \quad$ Weather data}

173 Daily maximum and minimum temperatures were lower in Colorado than in Florida (Supplementary

174 Figure S1, Supplementary Figure S2, and Supplementary Figure S3). Minimum day length during the

175 experimental period in Colorado was 12.05 hours in 2019 and 12.85 hours in 2020 while that was

17610.25 hours in Florida in 2018. Daylength was slowly decreasing from planting to flowering in all the

177 trials. In Colorado, the minimum daylength when the first plots had 50\% flowering was 13.9 hours

178 with an average temperature of $22.9^{\circ} \mathrm{C}$ in 2019 and 14.52 hours with average temperature of $22.8^{\circ} \mathrm{C}$ 

with average temperature of $26.6^{\circ} \mathrm{C}$.

\subsection{Genome wide association studies}

182

All SNP markers after filtering for MAF were used for GWAS. We identified 30 MTAs corresponding to 20 unique SNPs for days to flowering that explained 8-12\% of phenotypic variance in the GWAS conducted using four software in the three datasets (Table 2). These significant MTAs were distributed across seven chromosomes of the cowpea genome (Figure 2, Supplementary Figure S4-S6). In chromosome Vu03, FarmCPU identified a single SNP (2_03926). Multiple MTAs were identified on chromosome Vu04. FarmCPU, BLINK, and GLM identified the same significant SNP in chromosome Vu04 (2_55402), while both BLINK and GLM identified SNP 2_06977. GLM, FarmCPU, and BLINK further identified 7, 1, and 2 additional unique MTAs respectively, on chromosome Vu04 (Table 2). FarmCPU identified two unique MTAs (2_42453 and 2_43970) on chromosome Vu07. In chromosome Vu08, FarmCPU identified the same SNP (1_0362) in two studies (Colorado 2019 and Colorado 2020). FarmCPU further identified two unique MTAs in chromosome Vu09 and one unique MTA each in chromosome Vu10 and chromosome Vu11. BLINK identified one unique MTA in chromosome Vu10 (2_54017). MLM did not identify any significant MTAs in the three GWAS studies. Seven unique markers were reliable as they were identified by multiple algorithms or identified in more than 1 GWAS study (Table 2). In Colorado in 2019, early flowering alleles decreased flowering time by $5.50-6.93 \%$ corresponding to an average number of 4-6 days (Figure 3). Similarly, in Colorado in 2020, early flowering alleles decreased flowering time by 5.06-6.74\% corresponding to an average number of days to 4-5 days. In Florida in 2018, early flowering alleles decreased flowering time by $6.32 \%$ corresponding to a decrease in flowering by 3 days. 


\subsection{Candidate gene identification}

203 The linkage region of the 20 significant SNPs $(\mathrm{SNP} \pm 270 \mathrm{~kb})$ harbored a total of 483 unique gene

204 models on the cowpea genome. Functional annotation of these gene models using the Arabidopsis 205 gene network identified a total of 12 genes that were related to flowering (Table 3). These genes

206 included important genes like FLOWERING LOCUS T (FT), GIGANTEA (GI), Cryptochrome-2 207 (CRY2), LIGHT-DEPENDENT SHORT HYPOCOTYLS 3 (LSH3), REBELOTE (RBL) that are

208 known to control flowering time in Arabidopsis and other species (El-Assal et al., 2001; Teper-

209 Bamnolker and Samach, 2005; Prunet et al., 2008; Takeda et al., 2011; Park et al., 2020). These

210 candidate genes were located in chromosomes Vu04, Vu07, Vu08, and Vu09. In chromosome Vu04,

211 the peak signal at locus 2_46442 was associated with RBL gene, 2_55402 was associated with FT

212 gene, and 2_27454 was associated with GI gene. In chromosome Vu07, the peak signal at locus

213 2_42453 was associated with two genes $C R Y 2$ and $L S H 3$. In chromosome Vu08, locus 1_0362 was

214 tied to three genes: UGT87A2, BBX32 and Snf1 kinase interactor-like protein. Finally, in

215 chromosome Vu09, locus 2_39424 was associated with NGA1, DCL1, and LIF2 while locus 2_04844

216 was associated with HTA9.

\section{Discussion}

218 This study evaluated the variation in flowering time in the cowpea UCR Minicore in two contrasting

219 environments in Colorado and Florida. There was a wide variation in days to flower in all trials. We

220 observed high $H^{2}$ estimates (0.72-0.95) for flowering time in cowpea, which is similar to the

221 estimates reported in other species like soybean (0.77-0.95) (Zhang et al., 2015; Mao et al., 2017),

222 and alfalfa (0.38-0.75) (Adhikari et al., 2019). High $H^{2}$ of flowering time shows the inherent genetic

223 control of flowering as seen in other species. A $H^{2}$ of $84.5 \%$ was reported for days to flower in

224 cowpeas (Omoigui et al., 2006) and a narrow-sense heritability $\left(h^{2}\right)$ of $86 \%$ was reported in a cross 
bioRxiv preprint doi: https://doi.org/10.1101/2021.04.01.438123; this version posted April 2, 2021. The copyright holder for this preprint (which

between photoperiod-sensitive and photoperiod-insensitive varieties with at least seven major gene

pairs estimated to control time of flowering in this population (Ishiyaku et al., 2005). Since flowering

time is an important trait for plant breeders, the presence of variation in flowering time for cowpea

shows a large potential to manipulate its expression by breeding and selection.

229

230

231

232

233

234

235

236

237

238

239

240

241

242

243

244

245

Flowering time is a complex trait (Weller and Ortega, 2015) and is generally regulated by genetic networks composed of four main converging pathways: autonomous, gibberellin, photoperiod, and vernalization (Roux et al., 2006). These pathways integrate physiological and environmental cues to activate the transition from vegetative to reproductive stages at an optimum time (Brock et al., 2009). In Arabidopsis, induced mutations revealed the existence of up to 80 loci that affected flowering time (Levy and Deant, 1998). In cowpea, previous studies aimed at elucidating the genetics of flowering time have mostly focused on QTL analysis. Three QTLs related to days to flower and five QTLs related to time of flower opening were identified using 202 SSR markers in a mapping population of $159 \mathrm{~F}_{7}$ lines obtained by crossing a short duration variety (524B) to a long duration variety (219-01)

(Andargie et al., 2013). The linkage groups in this study were not named based on the reference genome (Lonardi et al., 2019), therefore, these QTLs could not be directly compared with our results. SNP and SSR markers were utilized in another RIL population of ZN016 $\times$ ZJ282 to identify QTLs for days to first flowering, nodes to first flower, leaf senescence, and pod number per plant (Xu et al., 2013). One major QTL and few minor QTLs were found to dominate each of the four traits with three to four QTLs controlling individual traits. Similarly, two QTLs on chromosome Vu05 and chromosome Vu09 with peak SNPs at 2_05332 (854,745 bp) and 2_03945 (5,449,874 bp)

respectively, were identified for days to flowering using $215 \mathrm{~F}_{8}$ RILs derived from a cross between cultivated (IT99K-573-1-1) and wild (TVNu-1158) cowpea accession (Lo et al., 2018). Studies on the cowpea multi-parent advanced generation intercross (MAGIC) population have identified flowering time loci with up to $25 \%$ phenotypic variability explained (PVE) and additive effect size of 
7 days under long-days but not under short-days (Olatoye et al., 2019). Drought tolerance index for

250 flowering time in this population identified significant SNPs (2_06470, 2_52919, 2_06137, and

251 1_0946) on chromosome Vu03 that were 12Mb downstream of the significant SNP identified in our

252 study (2_03926) (Ravelombola et al., 2021). Researchers have proposed one-gene (Sène, 1967) and

253 seven-gene (Ishiyaku et al., 2005) models to control flowering in cowpea and suggest that distinct

254 and common genetic regulators control flowering time adaptation to both long- and short-day

255 photoperiod in cowpea (Olatoye et al., 2019). Few GWAS have been reported in cowpea for pod

256 length (Xu et al., 2017), root architecture (Burridge et al., 2017), black seed coat color (Herniter et

257 al., 2018), and seed weight, length, width, and density (Lo et al., 2019). The availability of the

258 reference genome of cowpea and the Cowpea iSelect Consortium Array have opened up new avenues

259 in cowpea genetic analysis (Lonardi et al., 2019). The Cowpea iSelect Consortium Array with 51,128

260 SNPs is an excellent tool to identify marker trait associations and population genetic studies in

261 cowpea (Huang et al., 2018a).

262 For GWAS, we used four algorithms implemented in GAPIT (Lipka et al., 2012), namely, GLM,

263 MLM, FarmCPU (Liu et al., 2016), and BLINK (Huang et al., 2018b). In a GLM, false positives are

264 eliminated by fitting population structure as covariate (Price et al., 2006) and in MLM, population

265 structure and genetic effect of each individual is fitted as covariates (Yu et al., 2006). FarmCPU

266 performs marker tests with associated markers as covariates in a fixed effect model (Liu et al., 2016)

267 and assumes that quantitative trait nucleotides (QTN) underlying the trait are distributed equally

268 across the genome. Optimization on the associated covariate markers is done separately in a random

269 effect model. On the other hand, BLINK eliminates the requirement of equal distribution of QTNs by

270 taking linkage disequilibrium into consideration (Huang et al., 2018b). It also replaces the Restricted

271 Maximum Likelihood (REML) in the mixed linear model in FarmCPU with Bayesian Information 
272 Content (BIC) in a fixed effect model to boost computing speed. These algorithms identified multiple

273 MTAs for flowering time that were distributed in seven chromosomes in the cowpea genome.

274 Seven significant SNPs identified in our study harbored important flowering time related genes. On

275 chromosome Vu04, RBL gene was $197 \mathrm{~kb}$ upstream of the significant SNP (2_46442) and this gene

276 redundantly influences floral meristem termination (Prunet et al., 2008). FT was located $124 \mathrm{~kb}$

277 downstream of the most significant SNP (2_55402). FT, together with LEAFY (LFY), integrates

278 environmental signaling for induction of flowering (Moraes et al., 2019). Arabidopsis FT is a

279 member of a six-gene family that includes another important flowering-related gene, TERMINAL

280 FLOWER1 (TFL1) that delays transition to flowering and has been identified in legumes like pea,

281 Medicago, and lotus (Hecht et al., 2005). FT is expressed in leaves and is induced by long-day

282 treatment in Arabidopsis (Teper-Bamnolker and Samach, 2005). Additionally, in chromosome Vu04,

$283 G I$ was located $70 \mathrm{~kb}$ downstream of the most significant SNP (2_27454). GI-mediated integration of

284 photoperiodic and temperature information shapes thermo-morphogenic adaptation responses in

285 plants that optimizes plant growth and fitness in warm climates (Park et al., 2020). A total of 11

286 SNPs significantly associated with flowering time were identified in chromosome Vu04 showing that

287 this chromosome is very important in cowpea for adaptation and selection for flowering. On

288 chromosome Vu07, SNP 2_42453 harbored multiple genes. CRY2 was located $155 \mathrm{~kb}$ downstream of

289 the SNP while LSH3 was located $230 \mathrm{~kb}$ downstream of the SNP. CRY2 is a blue light receptor that

290 mediates blue-light regulated cotyledon expansion and is involved in the flowering response to

291 photoperiod in Arabidopsis (El-Assal et al., 2001). It is also a positive regulator of the flowering-time

292 gene CONSTANS (Guo et al., 1998). LSH3, also known as ORGAN BOUNDARY 1 encodes ALOG

293 family proteins and is expressed at the boundary of shoot apical meristem and lateral organs (Takeda

294 et al., 2011). Constitutive expression of LSH3 and LSH4 generates chimeric floral organs. 
located $137 \mathrm{~kb}$ upstream, and Snf1 kinase interactor-like protein located $231 \mathrm{~kb}$ downstream of the SNP. UGT87A2 promotes early flowering and is an important player in the autonomous pathway

(Wang et al., 2012) while $B B X 32$ is regulated by circadian clock and regulates flowering and modifications while breeding cowpea and further detailed studies of these candidate genes will help

311 MTAs in our study could not be directly compared to previous QTL studies (Andargie et al., 2013;

$312 \mathrm{Xu}$ et al., 2013) because of the absence of common markers. In a previous QTL study, two

313 significant QTLs for days to flowering were detected, one each on chromosome 5 and chromosome 9

314 that harbored phytochrome E and transcription factor TCP 18 that are involved in flowering time (Lo

315 et al., 2018). Similarly, another QTL report identified three QTLs related to days to flowering, one

316 each on LG1, LG2, and LG7 (Andargie et al., 2013). Our GWAS results detected significant reliable

317 SNPs on chromosome Vu04 and Vu08. A recent study that utilized the SNP array in the cowpea

318 UCR Minicore identified the same SNP (2_06977) on chromosome 4 under long days in California 
bioRxiv preprint doi: https://doi.org/10.1101/2021.04.01.438123; this version posted April 2, 2021. The copyright holder for this preprint (which

319 (Muñoz-Amatriaín et al., 2021). In our analysis, this SNP was identified by multiple algorithms in

320 two different datasets and is most likely an important region of interest for flowering time.

321 Interestingly, another study that utilized the SNP array identified two QTLs for flowering time in

322 chromosomes 5 and 9 that could explain 20-79\% of the phenotypic variance (Lo et al., 2018). On

323 chromosome 9, the previously identified QTL was 1.3 Mb upstream of the SNP (2_04844) identified

324 in this study. This suggests that these regions harbor important flowering related genes. Previous

325 studies reported that the QTLs could explain 5-18.5\% (Andargie et al., 2013), 16-30\% (Xu et al.,

326 2013), and 20-79\% (Lo et al., 2018) of the phenotypic variance for days to flowering depending on

327 the population. In our study, the variation explained by the MTAs varied from 8-12\%, indicating that

328 multiple genes might be affecting the traits and those genes have small effects. Our GWAS results in

329 Florida were limited to accessions that flowered under the long-day conditions of Riverside (CA,

330 USA) lines only, therefore, GWAS results from this location might miss some markers that were

331 identified in Colorado where the whole mini-core was evaluated. Nevertheless, our study contributes

332 with a large number of MTAs in cowpea for flowering time. Several loci identified here can be

333 further explored for use in marker-assisted selection, genomic selection, and gene discovery.

334 Plant breeders develop new varieties with increased yield by improving the crop's adaptability and

335 stress tolerance (Brummer et al., 2011). Flowering time has been associated with adaptation and

336 agronomic performance of traits in several crops. Early flowering plants could mature earlier and

337 avoid drought stress. Considerable gains can be made to increase yield and stability of grain legumes

338 in drought prone environments by shortening crop duration (Subbarao et al., 1995). This would be

339 important in Colorado and other regions of the semi-arid High Plains, where dryland agriculture

340 constitutes a significant proportion of the total cropland and where erratic precipitation patterns due

341 to climate change are threatening the productivity and profitability of such system (Rosenzweig and

342 Schipanski, 2019). Earlier flowering cowpea varieties could also help intensify dryland cropping 
343 systems in the High Plains by providing a viable alternative to the summer fallow that precedes

344 winter wheat (Nielsen and Vigil, 2005). In the case of Florida, although the Köppen-Trewartha

345 Climate Classification system has classified Central/North Florida as a Subtropical and

346 Mediterranean climate, and South Florida as a Tropical climate (Belda et al., 2014), drought stress is

347 a seasonal abiotic stressor in the state due to its sandy soil and high evaporative demand.

348 Early flowering can be transferred to cultivated cowpea through hybridization with early flowering

349 accessions. Selection of early flowering cowpea that performs well in subtropical regions will

350 undoubtedly help to increase the global production of cowpea as well as help to develop climate

351 resilient cowpea accessions. On the other hand, extended vegetative period in late maturing varieties

352 can provide higher biomass production which would be ideal for forage and cover crop cultivation,

353 where the crops can be terminated before they flower and seed, thus avoiding potential invasiveness.

354 Vegetative growth and rate of plant production have been shown to have additive and epistatic

355 relationships with flowering time QTLs in common beans using comparative QTL mapping,

356 suggesting pleiotropic effects between these traits (González et al., 2016). Further research is needed

357 to identify the haplotypes that confer early or late flowering trait in cowpeas. This study established

358 the basis for marker-assisted selection of flowering time in cowpea breeding programs. Additionally

359 the recent availability of the reference genome (Lonardi et al., 2019), development of the cowpea

360 UCR Minicore (Muñoz-Amatriaín et al., 2021), and future analysis of transcriptome profiles will

361 facilitate identification and manipulation of causative loci governing flowering time across a broad

362 range of environmental conditions.

\section{Figure legends}


364 Figure 1: Histogram of days to flower for the cowpea mini-core collection: (A) 367 accessions

365 planted in 2019 in Colorado; (B) 367 accessions planted in 2020 in Colorado; and (C) 292 accessions

366 planted in 2018 in Florida.

367 Figure 2: PhenoGram showing significant marker-trait associations for flowering time on each

368 chromosome. The grey bars within each chromosome show the locus of SNPs in the chromosome.

369 Each shape represents a significant SNP identified by the three algorithms (circle = BLINK, diamond

$370=$ FarmCPU, and triangle $=$ GLM). The color within each shape represents SNPs identified in the

371 different studies $($ blue $=$ Colorado 2019, green $=$ Colorado 2020, and red $=$ Florida 2018) .

372 Figure 3. Boxplot of days to flower as affected by the alleles present on the population (A) 367

373 accessions of the cowpea mini-core collection planted in 2019 in Colorado; and (B) 367 accessions

374 of the cowpea mini-core collection planted in 2020 in Colorado; and (C) 292 accessions of the

375 cowpea mini-core collection planted in 2018 in Florida.

\section{$376 \quad 6 \quad$ Table legends}

377 Table 1. Estimates of genotypic $\left(\mathrm{s}_{\mathrm{g}}^{2}\right)$ and residual $\left(\mathrm{s}_{\mathrm{e}}^{2}\right)$ variance components, broad-sense heritability

$378\left(H^{2}\right)$, standard error (SE) of the $H^{2}$, number of accessions planted, mean, and range for days to

379 flowering in the three studies.

380 Table 2. Significant SNPs related to days to flowering identified by multiple algorithms in genome

381 wide association studies in the three studies along with their $p$ value, minor allele frequency (MAF),

382 effect, percentage of variance explained $(\operatorname{PVE}(\%))$ as reported by each software, and $-\log _{10}(p)$.

383 Table 3 . Genes related to flowering time that are within $\pm 270 \mathrm{~kb}$ of the significant SNPs. Locus name

384 is the name of the gene in the cowpea reference genome with start and end for each gene in the 
chromosome, name of the associated SNP, position of the SNP, associated gene name, and locus ID

of the associated gene in Arabidopsis.

\section{Supplementary figure legends}

Supplementary Figure S1. Daily maximum (MaxT) and minimum (MinT) temperature and photoperiod (orange line) in Ft. Collins, CO during the trial in 2019.

Supplementary Figure S2. Daily maximum (MaxT) and minimum (MinT) temperature and photoperiod (orange line) in Ft. Collins, CO during the trial in 2020.

Supplementary Figure S3. Daily maximum (MaxT) and minimum (MinT) temperature and photoperiod (orange line) in Citra, FL during the trial in 2018. the cowpea mini-core collection planted in 2019 in Colorado. Left panel: Negative $\log _{10}$-transformed $\mathrm{P}$ values for each SNP (y axis) are plotted against the chromosomal position (y axis). The red line represents Bonferroni-corrected threshold of 0.05 for genome-wide statistically significant associations and the blue line shows suggestive associations $\left(p=1 \times 10^{-5}\right)$. Right panel shows the

399 QQ plots where $\mathrm{x}$-axis is expected negative log $\mathrm{p}$-values and the $\mathrm{y}$-axis is observed negative log $\mathrm{p}$ -

400 values. GWAS results for days to flowering using (A) BLINK; (B) FarmCPU; (C) GLM; and (D) 401 MLM.

403 the cowpea mini-core collection planted in 2020 in Colorado. Left panel: Negative $\log _{10}$-transformed

$404 \quad \mathrm{P}$ values for each SNP (y axis) are plotted against the chromosomal position (y axis). The red line

405 represents Bonferroni-corrected threshold of 0.05 for genome-wide statistically significant

406 associations and the blue line shows suggestive associations $\left(p=1 \times 10^{-5}\right)$. Right panel shows the 
407 QQ plots where $\mathrm{x}$-axis is expected negative log $\mathrm{p}$-values and the $\mathrm{y}$-axis is observed negative log $\mathrm{p}$ -

408 values. GWAS results for days to flowering using (A) BLINK; (B) FarmCPU; (C) GLM; and (D)

409 MLM.

410 Supplementary Figure S6: Manhattan plots from the GWAS analysis pertaining to 292 accessions of

411 the cowpea mini-core collection planted in 2018 in Florida. Left panel: Negative $\log _{10}$-transformed P

412 values for each SNP (y axis) are plotted against the chromosomal position (y axis). The red line

413 represents Bonferroni-corrected threshold of 0.05 for genome-wide statistically significant

414 associations and the blue line shows suggestive associations $\left(p=1 \times 10^{-5}\right)$. Right panel shows the

415 QQ plots where $\mathrm{x}$-axis is expected negative $\log \mathrm{p}$-values and the $\mathrm{y}$-axis is observed negative log $\mathrm{p}$ -

416 values. GWAS results for days to flowering using (A) BLINK; (B) FarmCPU; (C) GLM; and (D)

417 MLM.

$418 \quad 8 \quad$ Conflict of Interest

419 The authors declare that the research was conducted in the absence of any commercial or financial

420 relationships that could be construed as a potential conflict of interest.

4219 Author Contributions

422 ER conceived the project. ER and RD collected the phenotypic data in Florida. MM and JR collected

423 the phenotypic data in Colorado and provided the genotypic data. DP analysed the data and wrote the

424 manuscript. All authors reviewed the manuscript.

\section{$425 \quad 10 \quad$ Funding}

426 This research was partially funded by the Colorado Dry Bean Association Committee. This research

427 was funded by the United States Agency for International Development under Cooperative 
Agreement AID-OAA-A-15-00039, Appui à la Recherche et au Développement Agricole (AREA)

project, and by the USDA National Institute of Food and Agriculture, Hatch project 1018058.

\section{Acknowledgments}

431 We would like to thank Dr. Timothy J. Close and Dr. Philip A. Roberts for providing the seeds, and

432 Brooke Sayre-Chavez and Amanda Amsberry for their help in scoring days to flowering in Colorado.

433 The authors thank all the Forage Breeding and Genetics Lab members and staff at the University of

434 Florida Plant Science Research and Education Unit, Citra, FL for providing help for the field trial and 435 data collection.

\section{References}

\section{7}

Adhikari, L., Makaju, S. O., and Missaoui, A. M. (2019). QTL mapping of flowering time and biomass yield in tetraploid alfalfa (Medicago sativa L.). BMC Plant Biol. 19, 1-15. doi:10.1186/s12870-019-2020-7.

Andargie, M., Pasquet, R. S., Muluvi, G. M., and Timko, M. P. (2013). Quantitative trait loci analysis of flowering time related traits identified in recombinant inbred lines of cowpea (Vigna unguiculata). Genome 294, 289-294.

Ballester, P., Navarrete-Gómez, M., Carbonero, P., Oñate-Sánchez, L., and Ferrándiz, C. (2015). Leaf expansion in Arabidopsis is controlled by a TCP-NGA regulatory module likely conserved in distantly related species. Physiol. Plant. 155, 21-32. doi:10.1111/ppl.12327.

Bastow, R., and Dean, C. (2002). The molecular basis of photoperiodism. Biol. Rhythm Res. 37, 353380. doi:10.1016/S1534-5807(02)00296-4.

Belda, M., Holtanová, E., Halenka, T., and Kalvová, J. (2014). Climate classification revisited: From 
Berner, D., Carsky, R., Dashiell, K., Kling, J., and Manyong, V. (1996). A land management based approach to integrated Striga hermonthica control in sub-Saharan Africa. Outlook Agric. 25,

Brock, M. T., Stinchcombe, J. R., and Weinig, C. (2009). Indirect effects of FRIGIDA: Floral trait (co)variances are altered by seasonally variable abiotic factors associated with flowering time. $J$.

Brummer, C., Barber, W. T., Collier, S. M., Cox, T. S., Johnson, R., Murray, S. C., et al. (2011). Evol. Biol. 22, 1826-1838. doi:10.1111/j.1420-9101.2009.01794.x.

Burridge, J. D., Schneider, H. M., Huynh, B. L., Roberts, P. A., Bucksch, A., and Lynch, J. P. (2017). Genome-wide association mapping and agronomic impact of cowpea root architecture. Theor. Appl. Genet. 130, 419-431. doi:10.1007/s00122-016-2823-y.

464 Craufurd, P. Q., Qi, A., Summerfield, R. J., Ellis, R. H., and Roberts, E. H. (1996). Development in version 4. Brisbane: The State of Queensland, Department of Primary Industries and Fisheries. cowpea (Vigna unguiculata). III. Effects of temperature and photoperiod on time to flowering in photoperiod-sensitive genotypes and screening for photothermal responses. Exp. Agric. 32, 29and agronomic traits in Florida. Submitt. Publ., 1-18. 
Durand, E., Bouchet, S., Bertin, P., Ressayre, A., Jamin, P., Charcosset, A., et al. (2012). Flowering time in maize: linkage and epistasis at a major effect locus. Genetics 190, 1547-1562. QTL for flowering time in Arabidopsis reveals a novel allele of CRY2. Nat. Genet. 29, 435-40.

Fatokun, C., Girma, G., Abberton, M., Gedil, M., Unachukwu, N., Oyatomi, O., et al. (2018). unguiculata (L.) Walp.) germplasm collection. Sci. Rep. 8, 16035.

González, A. M., Yuste-Lisbona, F. J., Saburido, S., Bretones, S., de Ron, A. M., Lozano, R., et al. (2016). Major contribution of flowering time and vegetative growth to plant production in common bean as deduced from a comparative genetic mapping. Front. Plant Sci. 7.

Guo, H., Yang, H., Mockler, T. C., and Lin, C. (1998). Regulation of flowering time by Arabidopsis doi:10.3389/fpls.2016.01940. 348. doi:10.1007/978-1-4614-8370-0. doi:10.1104/pp.104.057018. 
Herniter, I. A., Muñoz-Amatriaín, M., Lo, S., Guo, Y. N., and Close, T. J. (2018). Identification of candidate genes controlling black seed coat and pod tip color in cowpea (Vigna unguiculata [L.] Walp). G3 Genes, Genomes, Genet. 8, 3347-3355. doi:10.1534/g3.118.200521.

Huang, M., Liu, X., Zhou, Y., Summers, R. M., and Zhang, Z. (2018b). BLINK: A package for the next level of genome-wide association studies with both individuals and markers in the millions. Gigascience 8, 1-12. doi:10.1093/gigascience/giy154.

Huynh, B.-L., Close, T. J., Roberts, P. A., Hu, Z., Wanamaker, S., Lucas, M. R., et al. (2013). Gene pools and the genetic architecture of domesticated cowpea. Plant Genome 6, 1-8. doi:10.3835/plantgenome2013.03.0005.

Ishiyaku, M. F., Singh, B. B., and Craufurd, P. Q. (2005). Inheritance of time to flowering in cowpea

Jarillo, J. A., and Piñeiro, M. (2015). H2A.Z mediates different aspects of chromatin function and modulates flowering responses in Arabidopsis. Plant J. 83, 96-109. doi:10.1111/tpj.12873. (Vigna unguiculata (L.) Walp.). Euphytica 142, 291-300. doi:10.1007/s10681-005-2435-0.

\section{Kumar, J., and Abbo, S. (2001). Genetics of flowering time in chickpea and its bearing on} productivity in semiarid environments. Adv. Agron. 72, 107-138. 
513 Latrasse, D., Germann, S., Houba-Hérin, N., Dubois, E., Bui-Prodhomme, D., Hourcade, D., et al.

514 (2011). Control of flowering and cell fate by LIF2, an RNA binding partner of the polycomb complex component LHP1. PLoS One 6. doi:10.1371/journal.pone.0016592.

Levy, Y. Y., and Deant, C. (1998). Control of flowering time. Curr. Opin. Plant Biol. 1, 49-54.

517 Lipka, A. E., Tian, F., Wang, Q., Peiffer, J., Li, M., Bradbury, P. J., et al. (2012). GAPIT: Genome association and prediction integrated tool. Bioinformatics 28, 2397-2399.

Liu, X., Huang, M., Fan, B., Buckler, E. S., and Zhang, Z. (2016). Iterative usage of fixed and random effect models for powerful and efficient genome-wide association studies. PLoS Genet., 1-24. doi:doi:10.1371/journal.pgen.1005767.

Lo, S., Muñoz-Amatriaín, M., Hokin, S. A., Cisse, N., Roberts, P. A., Farmer, A. D., et al. (2019). A genome-wide association and meta-analysis reveal regions associated with seed size in cowpea [Vigna unguiculata (L.) Walp]. Theor. Appl. Genet. 132, 3079-3087. doi:10.1007/s00122-01903407-z. genome of cowpea (Vigna unguiculata [L.] Walp.). Plant J. 98, 767-782. doi:10.1111/tpj.14349.

Mao, T., Li, J., Wen, Z., Wu, T., Wu, C., Sun, S., et al. (2017). Association mapping of loci 
controlling genetic and environmental interaction of soybean flowering time under various photo-thermal conditions. BMC Genomics 18, 1-17. doi:10.1186/s12864-017-3778-3.

536

537

538

539

540

541

542

543

544

Martins, L. M. V., Xavier, G. R., Rangel, F. W., Ribeiro, J. R. A., Neves, M. C. P., Morgado, L. B., et al. (2003). Contribution of biological nitrogen fixation to cowpea: A strategy for improving grain yield in the semi-arid region of Brazil. Biol. Fertil. Soils 38, 333-339. doi:10.1007/s00374-003-0668-4.

Moraes, T. S., Dornelas, M. C., and Martinelli, A. P. (2019). FT/TFL1: Calibrating plant architecture. Front. Plant Sci. 10, 1-6. doi:10.3389/fpls.2019.00097.

Muñoz-Amatriaín, M., Lo, S., Herniter, I. A., Boukar, O., and Fatokun, C. (2021). The UCR Minicore : a valuable resource for cowpea research and breeding. bioRxiv, 1-18.

Muñoz-amatriaín, M., Lo, S., Herniter, I., Fatokun, C., Boukar, O., Guo, Y.-N., et al. Genetic characterization of the cowpea UCR Mini- Core brings insights into the genetic architecture of important agronomic traits. (in prep).

Muñoz-Amatriaín, M., Mirebrahim, H., Xu, P., Wanamaker, S. I., Luo, M. C., Alhakami, H., et al. (2017). Genome resources for climate-resilient cowpea, an essential crop for food security. Plant J. 89, 1042-1054. doi:10.1111/tpj.13404.

Nielsen, D. C., and Vigil, M. F. (2005). Legume green fallow effect on soil water content at wheat planting and wheat yield. Agron. J. 97, 684-689. doi:10.2134/agronj2004.0071.

Olatoye, M. O., Hu, Z., and Aikpokpodion, P. O. (2019). Epistasis detection and modeling for genomic selection in cowpea (Vigna unguiculata L. Walp.). Front. Genet. 10, 1-14. doi:10.3389/fgene.2019.00677. 
Omoigui, L. O., Ishiyaku, M. F., Kamara, A. Y., Alabi, S. O., and Mohammed, S. G. (2006). Genetic variability and heritability studies of some reproductive traits in cowpea (Vigna unguiculate (L.)

Park, Y. J., Kim, J. Y., Lee, J. H., Lee, B. D., Paek, N. C., and Park, C. M. (2020). GIGANTEA Walp.). African J. Biotechnol. 5, 1191-1195. doi:10.5897/AJB2006.000-5058.

561

562 shapes the photoperiodic rhythms of thermomorphogenic growth in Arabidopsis. Mol. Plant 13, 459-470. doi:10.1016/j.molp.2020.01.003.

Price, A. L., Patterson, N. J., Plenge, R. M., Weinblatt, M. E., Shadick, N. A., and Reich, D. (2006). Principal components analysis corrects for stratification in genome-wide association studies. Nat. Genet. 38, 904-909. doi:10.1038/ng1847.

Prunet, N., Morel, P., Thierry, A. M., Eshed, Y., Bowman, J. L., Negrutiu, I., et al. (2008). REBELOTE, SQUINT, and ULTRAPETALAl function redundantly in the temporal regulation of floral meristem termination in Arabidopsis thaliana. Plant Cell 20, 901-919. doi:10.1105/tpc.107.053306.

R Core Team (2018). R: A language and environment for statistical computing. R Found. Stat. Comput. doi:10.1007/978-3-540-74686-7.

Ravelombola, W., Shi, A., and Huynh, B.-L. (2021). Loci discovery, network-guided approach, and genomic prediction for drought tolerance index in a multi-parent advanced generation intercross (MAGIC) cowpea population. Hortic. Res. 8. doi:10.1038/s41438-021-00462-w.

Rodrigues, A. C., Silveira, J. A. G., Bonifacio, A., and Figueiredo, M. do V. B. (2013). Metabolism of nitrogen and carbon: Optimization of biological nitrogen fixation and cowpea development. Soil Biol. Biochem. 67, 226-234. doi:10.1016/j.soilbio.2013.09.001. 
Rosenzweig, S. T., and Schipanski, M. E. (2019). Landscape-scale cropping changes in the High

Roux, F., Touzet, P., Cuguen, J., and Le Corre, V. (2006). How to be early flowering: an doi:10.1088/1748-9326/ab5e8b.

Schmitz, R. J., Hong, L., Fitzpatrick, K. E., and Amasino, R. M. (2007). DICER-LIKE 1 and DICER-

Sène, D. (1967). Déterminisme génétique de la précocité chez Vigna unguiculata (L.) Walp.

Singh, B. (2020). Cowpea: the food legume of the 21st century. John Wiley \& Sons.

Subbarao, G. V., Johansen, C., Slinkard, A. E., Nageswara Rao, R. C., Saxena, N. P., and Chauhan, Y. S. (1995). Strategies for improving drought resistance in grain legumes. CRC. Crit. Rev. Plant Sci. 14, 469-523. doi:10.1080/07352689509701933.

Takeda, S., Hanano, K., Kariya, A., Shimizu, S., Zhao, L., Matsui, M., et al. (2011). CUP-SHAPED COTYLEDON1 transcription factor activates the expression of $L S H 4$ and $L S H 3$, two members cowpea Res. 10, 313-325. 
Teper-Bamnolker, P., and Samach, A. (2005). The flowering integrator FT regulates SEPALLATA3 and FRUITFULL accumulation in Arabidopsis leaves. Plant Cell 17, 2661-2675.

Tripathi, P., Carvallo, M., Hamilton, E. E., Preuss, S., and Kay, S. A. (2017). Arabidopsis B-BOX32 interacts with CONSTANS-LIKE3 to regulate flowering. Proc. Natl. Acad. Sci. U. S. A. 114, doi:10.1105/tpc.105.035766.

Turner, S. D. (2014). qqman: an R package for visualizing GWAS results using Q-Q and manhattan plots. bioRxiv, 1-2. doi:https://doi.org/10.1101/005165.

Wang, B., Jin, S. H., Hu, H. Q., Sun, Y. G., Wang, Y. W., Han, P., et al. (2012). UGT87A2, an Arabidopsis glycosyltransferase, regulates flowering time via FLOWERING LOCUS C. New Phytol. 194, 666-675. doi:10.1111/j.1469-8137.2012.04107.x.

Xiong, H., Shi, A., Mou, B., Qin, J., Motes, D., Lu, W., et al. (2016). Genetic diversity and population structure of cowpea (Vigna unguiculata L. walp). PLoS One 11, 1-15.

Weller, J. L., and Ortega, R. (2015). Genetic control of flowering time in legumes. Front. Plant Sci. 6, 182-188. doi:10.3389/fpls.2015.00207.

613 Xu, P., Wu, X., Muñoz-Amatriaín, M., Wang, B., Wu, X., Hu, Y., et al. (2017). Genomic regions, cellular components and gene regulatory basis underlying pod length variations in cowpea ( $V$.

616 Xu, P., Wu, X., Wang, B., Hu, T., Lu, Z., Liu, Y., et al. (2013). QTL mapping and epistatic 617 interaction analysis in asparagus bean for several characterized and novel horticulturally 
Yu, J., and Buckler, E. S. (2006). Genetic association mapping and genome organization of maize. Curr. Opin. Biotechnol. 17, 155-60. doi:10.1016/j.copbio.2006.02.003. mixed-model method for association mapping that accounts for multiple levels of relatedness. Nat. Genet. 38, 203-208. doi:10.1038/ng1702.

\section{Data Availability Statement}

The genetic data used in this study is available at (Muñoz-Amatriaín et al., 2021).

\section{Contribution to the Field Statement}

630 Plant adaptation to new climates and their yield are strongly affected by flowering time. Early

631 flowering plants could mature earlier and avoid drought stress. This might be a good adaptation

632 strategy to cope with impending climate change crisis, especially in regions with lower access to

633 irrigation water. Therefore, understanding the genetic basis of flowering time is critical to advance

634 plant breeding. Genome wide association studies for flowering time have been done in other species,

635 however, this has not been widely reported in cowpea. Cowpea is highly heat tolerant and is an

636 important crop to breed for new varieties that are resilient to changing climates. Cowpea is a major

637 source of protein for human consumption as well as a quality forage for animal feed. To facilitate

638 future plant breeding efforts, we have identified marker trait associations related to flowering time in 639 a cowpea mini-core collection. This study contributed large number of marker trait associations in 
640 cowpea for flowering time and identified candidate genes related to flowering time in cowpea.

641 Several loci identified here can be validated in other populations to support cowpea breeding

642 programs with introgression of favorable alleles and marker-assisted selection, genomic selection,

643 and gene discovery. To our knowledge, this is the first published study that has done GWAS for

644 flowering time in cowpea using the cowpea mini-core collection. 
(A)

Colorado 2019

(B)

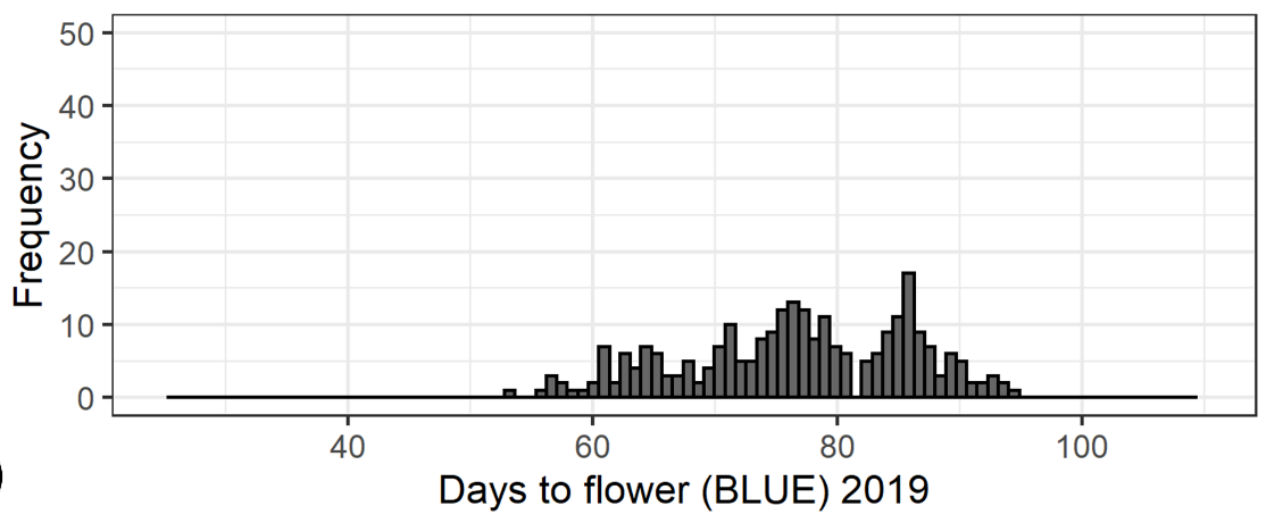

Colorado 2020

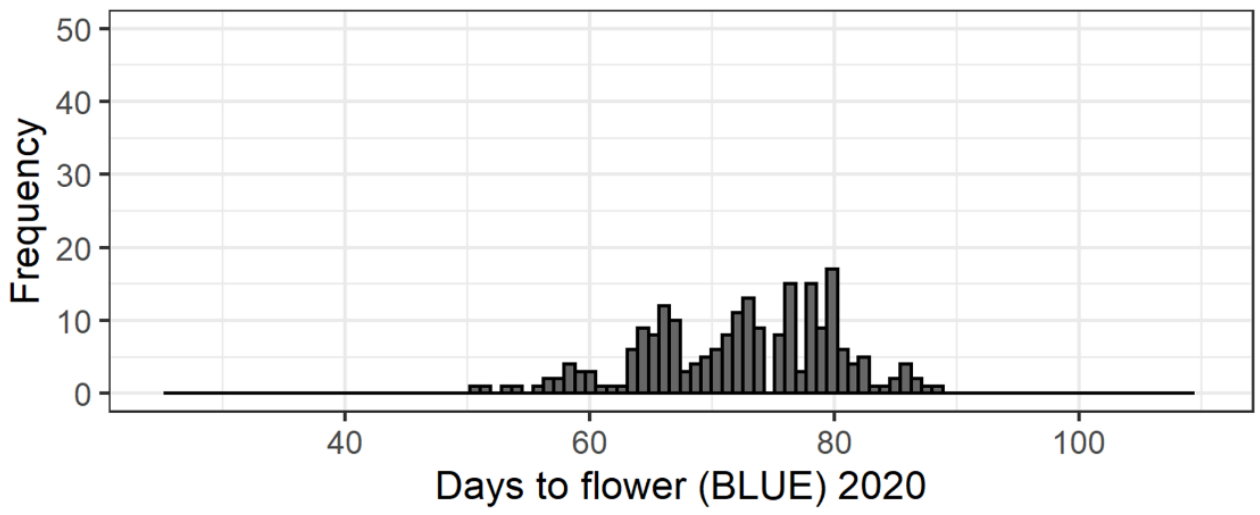

(C)
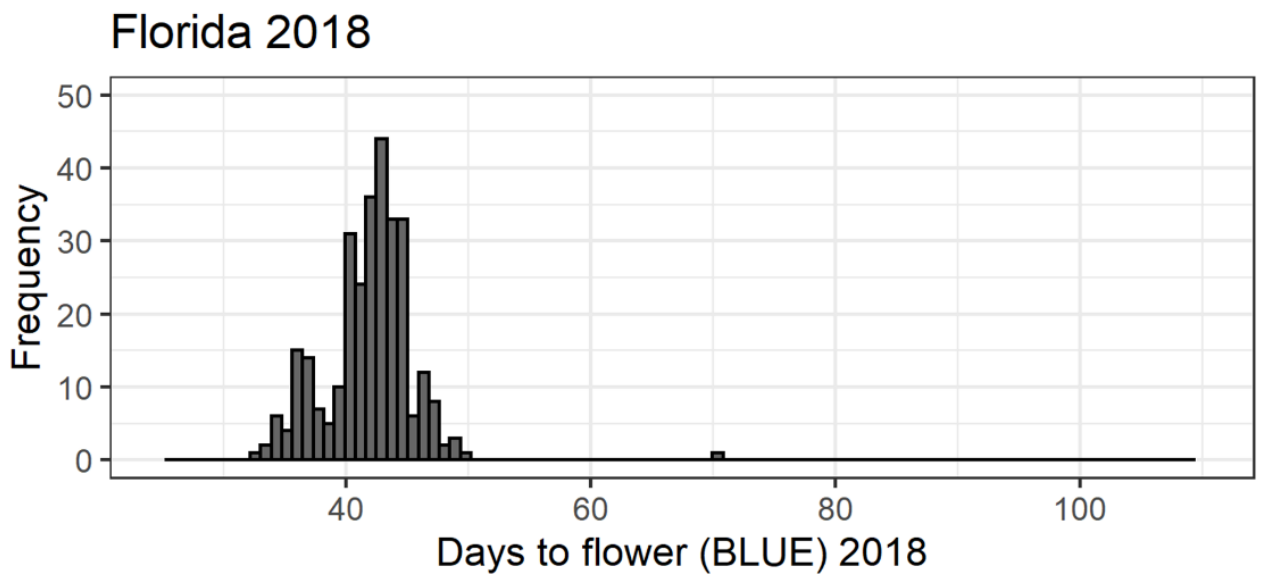

646 Figure 1: Histogram of Days to flower for: (A) 367 accessions of the cowpea mini-core collection

647 planted in 2019 in Colorado; (B) 367 accessions of the cowpea mini-core collection planted in 2020

648 in Colorado; and (C) 292 accessions of the cowpea mini-core collection planted in 2018 in Florida. 


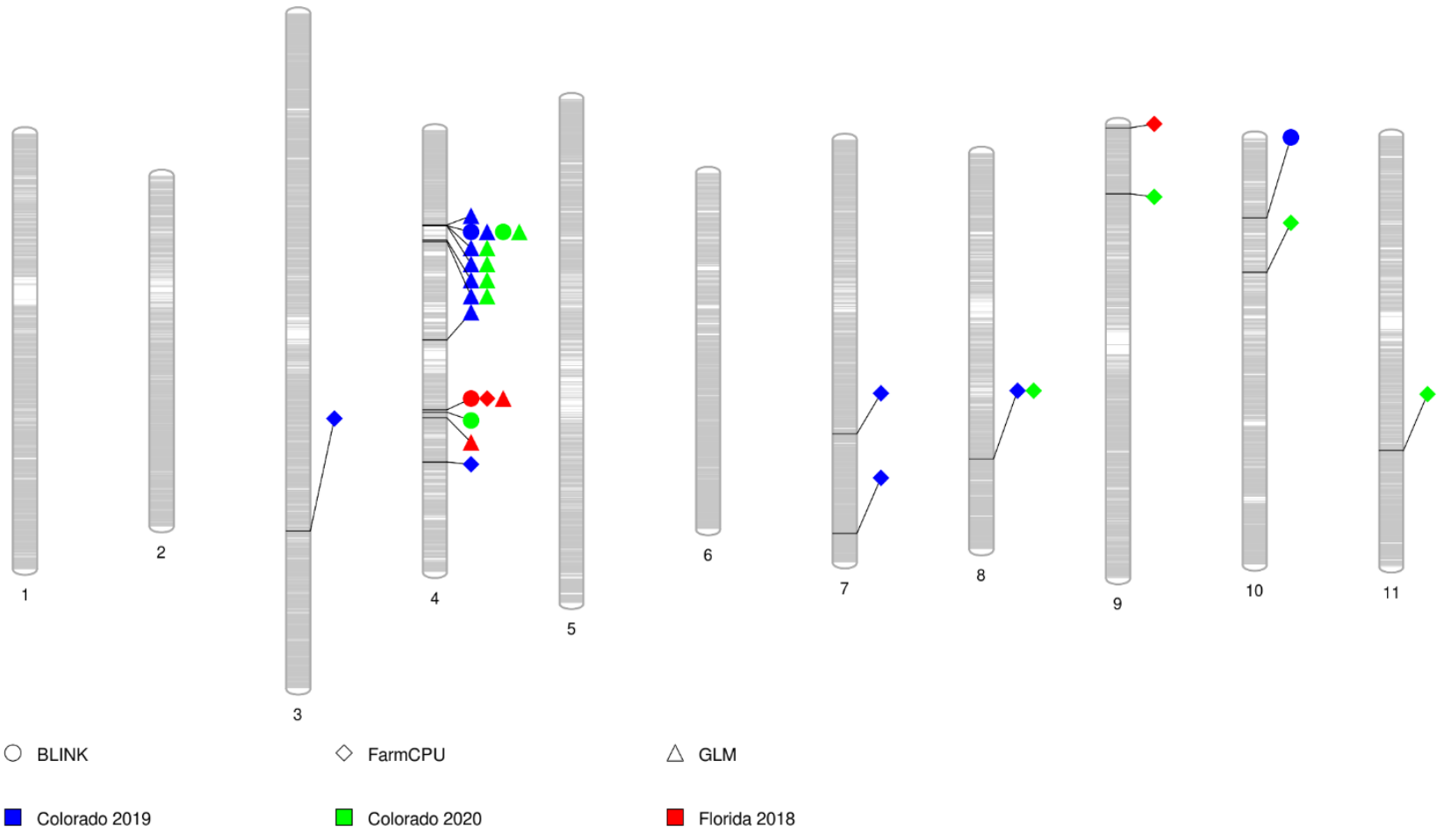

651 Figure 2: PhenoGram showing significant marker-trait associations for flowering time on each

652 chromosome. The grey bars within each chromosome show the locus of SNPs in the chromosome.

653 Each shape represents a significant SNP identified by the three algorithms $($ circle $=$ BLINK, diamond

$654=$ FarmCPU, and triangle $=$ GLM). The color within each shape represents SNPs identified in the

655 different studies $($ red $=$ Florida 2018, blue $=$ Colorado 2019, and green $=$ Colorado 2020 $)$. 
(A)

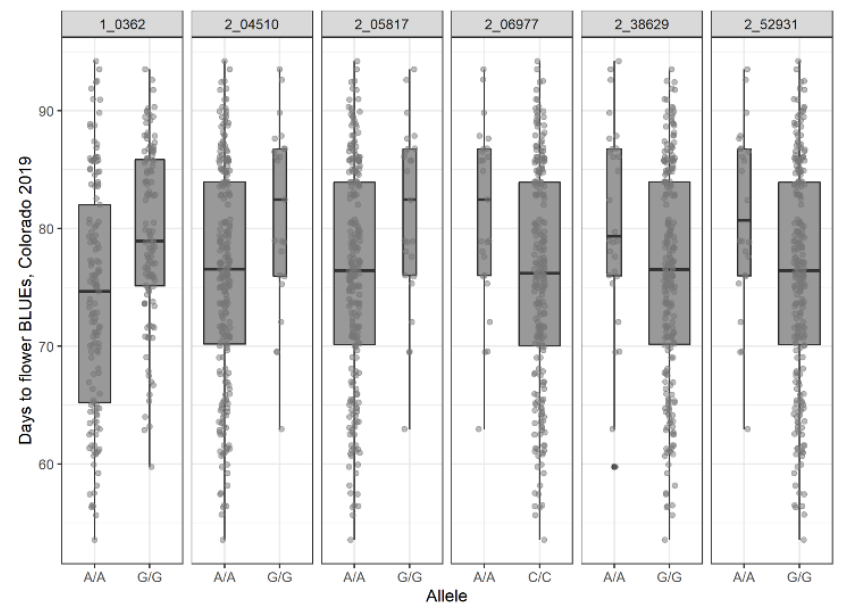

(B)

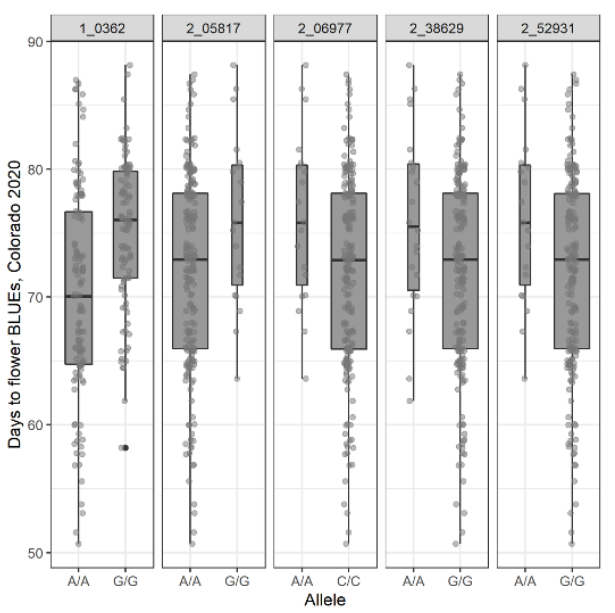

(C)

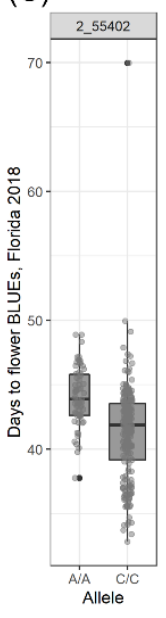

659 Figure 3. Boxplot of days to flower as affected by the alleles present on the population (A) 367

660 accessions of the cowpea mini-core collection planted in 2019 in Colorado; (B) 367 accessions of the

661 cowpea mini-core collection planted in 2020 in Colorado; and (C) 292 accessions of the cowpea

662 mini-core collection planted in 2018 in Florida. 
663 Table 1. Estimates of genotypic $\left(\mathrm{s}^{2} \mathrm{~g}\right)$ and residual $\left(\mathrm{s}_{\mathrm{e}}^{2}\right)$ variance components, broad-sense heritability

$664\left(H^{2}\right)$, standard error (SE) of the $H^{2}$, number of accessions planted, mean, and range for days to

665 flowering in the three studies.

\begin{tabular}{rrrrrrrr}
\hline Location & Year & $\begin{array}{c}\text { Accessions } \\
\text { Evaluated }\end{array}$ & Mean & Range & $\boldsymbol{H}^{2}+\mathbf{S E}$ & $\mathbf{S}^{\mathbf{2}}$ & $\mathbf{S}^{\mathbf{2}}$ \\
\hline Colorado & 2019 & 367 & 75 & $56-100$ & $0.95 \pm 0.04$ & $102.62^{* * * *}$ & 4.07 \\
Colorado & 2020 & 367 & 72 & $50-88$ & $0.80 \pm 0.12$ & $47.62^{*}$ & 11.01 \\
Florida & 2018 & 292 & 41 & $32-69$ & $0.72 \pm 0.06$ & $11.01 * * *$ & 2.93
\end{tabular}

666 '***' denotes significance at $p<0.001$ and '*' denotes significance at $p<0.05$ for the Likelihood Ratio 667 Tests (LRT)

668 
670 Table 2. Significant SNPs related to days to flowering identified by multiple algorithms in genome

671 wide association studies in the three studies along with their $p$ value, minor allele frequency (MAF),

672 effect, percentage of variance explained $(\operatorname{PVE}(\%))$ as reported by each software, $\operatorname{and}-\log _{10}(p)$.

\begin{tabular}{|c|c|c|c|c|c|c|c|c|c|c|}
\hline SNP & Chr. & Position & $\underline{p \_ \text {value }}$ & MAF & Efect & Location & Year & Software & PVE (\%) & $-\log _{10}(p)$ \\
\hline 2_03926 & u03 & 50079486 & $7.10 \mathrm{E}-07$ & 0.22 & -2.36 & Colorado & 2019 & FarmCPU & NA & 6.15 \\
\hline 2_33309 & $\mathrm{V}$ & 9183563 & $2.34 \mathrm{E}-07$ & 0.11 & -5.00 & Colo & 2019 & GLM & 9.20 & 6.63 \\
\hline 2_06977 & & 9211195 & $2.68 \mathrm{E}-14$ & 0.11 & NA & Cols & 2019 & BLINK & NA & 13.57 \\
\hline 2_06977 & & 9211195 & $3.28 \mathrm{E}-09$ & 0.11 & -5.98 & Colorado & 2019 & GLM & 12.25 & 8.48 \\
\hline 2_06977 & Vu04 & 9211195 & $5.37 \mathrm{E}-12$ & 0.09 & NA & Colorado & 2020 & BLINK & NA & 11.27 \\
\hline 2_06977 & Vu04 & 9211195 & $8.24 \mathrm{E}-08$ & 0.09 & -4.94 & Colorado & 2020 & GLM & 11.32 & 7.08 \\
\hline 2_52931 & & 9224808 & 4.13E-08 & 0.09 & -5.62 & Colorado & 2019 & GLM & 10.42 & 7.38 \\
\hline 2_52931 & & 9224808 & $1.03 \mathrm{E}-07$ & 0.09 & -4.89 & Colorado & 2020 & GLM & 11.13 & 6.99 \\
\hline 2_05817 & V & 9263427 & $3.13 \mathrm{E}-08$ & 0.10 & 5.61 & Colorado & 2019 & GLM & 10.62 & 7.50 \\
\hline 2_05817 & $\mathrm{VuO}$ & 9263427 & $1.53 \mathrm{E}-07$ & 0.09 & 4.84 & Colorado & 2020 & GLM & 10.81 & 6.81 \\
\hline 2_04510 & $\mathrm{V}$ & 10681090 & $5.14 \mathrm{E}-08$ & 0.09 & 5.69 & Color & 2019 & GLM & 10.27 & 7.29 \\
\hline 2_04510 & $v$ & 10681090 & 5.64E-07 & 0.08 & 4.71 & Colorado & 2020 & GLM & 9.77 & 6.25 \\
\hline 2_38629 & $\mathrm{V}$ & 1077 & $1.09 \mathrm{E}$ & 0 . & 4 & $\mathrm{C}$ & 19 & GLM & 2 & 5.96 \\
\hline $2 \_38629$ & V & 10776312 & $1.04 \mathrm{E}-06$ & 0.1 & -4.14 & $\mathrm{Col}$ & 2020 & GI & 9.28 & 5.98 \\
\hline 2_46442 & $\mathrm{V}$ & 20308708 & $1.01 \mathrm{E}-06$ & 0.38 & 3.17 & Colorado & 2019 & GLM & 8.18 & 6.00 \\
\hline 2_55402 & & 27032485 & $3.10 \mathrm{E}-11$ & 0.25 & -1.16 & Florida & 2018 & FarmCPU & NA & 10.51 \\
\hline 2_55402 & & 2703 & $3.65 \mathrm{E}-13$ & 0.25 & NA & Florida & 2018 & BLINK & NA & 12.44 \\
\hline 2_55402 & V & 2703 & $2.40 \mathrm{~F}$ & 0 & -1.41 & & 8 & & 10 & 7.62 \\
\hline 2_52369 & & 27310629 & $2.93 \mathrm{E}-07$ & 0.33 & NA & $\mathrm{Col}$ & 2020 & BLINK & NA & 6.53 \\
\hline $2 \_22451$ & & 27793336 & 4.77E-07 & 0.18 & 1.45 & Florida & 2018 & GLM & 8.15 & 6.32 \\
\hline $2 \_27454$ & & 32104992 & $4.58 \mathrm{E}-07$ & 0.29 & 2.31 & Colorado & 2019 & FarmCPU & NA & 6.34 \\
\hline 2_42453 & & 2848 & $3.75 \mathrm{~F}$ & & -2.2 & Color & & & $\mathrm{N}$ & 6.43 \\
\hline $2 \_43$ & Vu07 & & 6.80 & & -2.76 & & & & $\mathrm{~N}$ & 6.17 \\
\hline 1_0362 & Vu08 & 29639172 & $2.61 \mathrm{E}-07$ & 0.45 & 1.83 & Colorado & 2019 & FarmCPU & NA & 6.58 \\
\hline 1_0362 & Vu08 & 29639172 & 7.07E-07 & 0.45 & 1.47 & Colorado & 2020 & FarmCPU & NA & 6.15 \\
\hline 2_39424 & Vu09 & 9806 & $1.62 \mathrm{E}-08$ & 0 . & -0.79 & Florid & 2018 & FarmCPU & NA & 7.79 \\
\hline 2_04844 & Vu09 & 6752951 & $9.22 \mathrm{E}-11$ & 0.27 & 2.97 & Colorado & 20 & nCPU & NA & 10.04 \\
\hline 2_54017 & Vu10 & 7807120 & $2.99 \mathrm{E}-08$ & 0.26 & NA & Colorado & 2019 & BLINK & NA & 7.52 \\
\hline 2_42049 & Vu10 & 13068722 & 3.92E-08 & 0.12 & 4.76 & Colorado & 2020 & FarmCPU & NA & 7.41 \\
\hline 2_03469 & Vu11 & 30442337 & $4.48 \mathrm{E}-07$ & 0.18 & -1.96 & Colorado & 2020 & FarmCPU & NA & 6.35 \\
\hline
\end{tabular}

673 
676 Table 3 . Genes related to flowering time that are within $\pm 270 \mathrm{~kb}$ of the significant SNPs. Locus name

677 is the name of the gene in the cowpea reference genome with start and end for each gene in the

678 chromosome, name of the associated SNP, position of the SNP, associated gene name, and locus ID

679 of the associated gene in Arabidopsis.

\begin{tabular}{|c|c|c|c|c|c|c|c|}
\hline Locus Name & Chr & Start & End & SNP & Position & $\begin{array}{l}\text { Gene } \\
\text { Name }\end{array}$ & Arabidopsis locus ID \\
\hline Vigun04g096400 & Vu04 & 20505963 & 20516534 & 2_46442 & 20308708 & $R B L$ & AT3G55510 \\
\hline Vigun04g109500 & Vu04 & 27156677 & 27161340 & 2_55402 & 27032485 & $F T$ & AT1G65480 \\
\hline Vigun04g126700 & Vu04 & 32034709 & 32050305 & 2_27454 & 32104992 & $G I$ & AT1G22770 \\
\hline Vigun07g171300 & Vu07 & 28639274 & 28644217 & $2 \_42453$ & 28483321 & $C R Y 2$ & AT1G04400 \\
\hline Vigun07g171900 & Vu07 & 28713837 & 28716092 & 2_42453 & 28483321 & LSH3 & AT2G31160 \\
\hline Vigun08g124100 & Vu08 & 29426933 & 29428985 & 1_0362 & 29639172 & UGT87A2 & AT2G30140 \\
\hline Vigun08g127400 & Vu08 & 29776374 & 29777564 & 1_0362 & 29639172 & BBX32 & AT3G21150 \\
\hline Vigun08g128600 & Vu08 & 29870661 & 29873299 & 1_0362 & 29639172 & Snfl kinase & AT1G80940 \\
\hline Vigun09g003600 & Vu09 & 249165 & 253046 & 2_39424 & 419806 & $N G A 1$ & AT2G46870 \\
\hline Vigun09g003800 & Vu09 & 275035 & 288027 & 2_39424 & 419806 & $D C L 1$ & AT1G01040 \\
\hline Vigun09g005800 & Vu09 & 426595 & 430905 & 2_39424 & 419806 & $L I F 2$ & AT4G00830 \\
\hline Vigun09g063700 & Vu09 & 6692636 & 6694999 & 2_04844 & 6752951 & HTA9 & AT1G52740 \\
\hline
\end{tabular}

